\title{
Traduire
}

Ine autre perspective sur la tataduction

Revue française de la traduction

$236 \mid 2017$

Traducteurs en blouse blanche

\section{Essai d'analyse socioterminologique et contrastive de textes de loi algériens}

\section{Nourredine Bessadi}

\section{(2) OpenEdition}

1 Journals

Édition électronique

URL : http://journals.openedition.org/traduire/927

DOI : $10.4000 /$ traduire.927

ISSN : 2272-9992

Éditeur

Société française des traducteurs

Édition imprimée

Date de publication : 1 juin 2017

Pagination : 85-91

ISSN : 0395-773X

\section{Référence électronique}

Nourredine Bessadi, «Essai d'analyse socioterminologique et contrastive de textes de loi algériens »,

Traduire [En ligne], 236 | 2017, mis en ligne le 01 juin 2019, consulté le 10 décembre 2020. URL : http:// journals.openedition.org/traduire/927 ; DOI : https://doi.org/10.4000/traduire.927 


\section{Essai d'analyse socioterminologique et contrastive de textes de loi algériens}

\section{Nourredine Bessadi}

\section{Introduction}

La communication est d'une importance capitale dans la prise en charge efficace des justiciables. Dans tout pays, le justiciable, souvent non-initié, et le professionnel du droit doivent communiquer efficacement. Or, en Algérie, des difficultés apparaissent fréquemment en raison du caractère plurilingue de la société algérienne. En effet, si le professionnel du droit est formé en arabe, les textes officiels de la République algérienne sont toujours rédigés en deux langues: arabe et français. Cette particularité produit ce que l'on appelle un bruit au niveau du code : une entrave à la transmission normale du message due au choix des signes linguistiques ou au non-respect des règles de fonctionnement de la langue. II arrive aussi que l'encodage soit réussi mais que le décodage échoue, le choix du code étant souvent contraint par les situations de communication.

Afin de contribuer à l'amélioration de cette situation, nous proposons ici de comparer la terminologie juridique française et arabe dans les textes officiels. Nous avons choisi les Codes algériens de la famille et de la nationalité car ils constituent la rencontre par excellence du droit musulman et de la tradition civiliste, via l'arabe et le français. Ainsi, en Algérie, deux cultures juridiques cohabitent : la culture juridique civiliste et la culture juridique musulmane, ancrées dans deux systèmes linguistiques très différents : le français, langue indo-européenne dont les descriptions grammaticales s'inscrivent profondément dans une tradition occidentale marquée par la philosophie aristotélicienne, et l'arabe, langue sémitique dont la tradition grammaticale reflète une pensée linguistique largement déterminée par l'interprétation du texte sacré, le Coran.

Nous proposons ici une comparaison des terminologies juridiques de ces deux langues puis une analyse contrastive des situations concrètes qui découlent de la traduction des textes officiels algériens. 


\section{Analyse socioterminologique}

Le statut de la langue arabe est particulier. Étroitement liée au Coran, elle reste pour certains la langue de la parole divine et de ce fait, la seule à pouvoir préserver la sacralité des termes issus du droit musulman dont elle subit fortement l'influence. Toute traduction serait donc une sorte d'altération de cette dimension sacrale.

Par ailleurs, le droit est un phénomène local qui franchit difficilement les frontières. II est de ce fait très imprégné de la langue et de la culture qu'elle porte, des us et coutumes et de l'histoire de la communauté sociale qui l'a vu naître et évoluer. La langue détermine souvent l'appartenance à tel ou tel système juridique et celui-ci, à son tour, marque de son sceau la langue qui le véhicule. Cela n'est évidemment pas sans conséquences. Le fait que le système juridique influence la langue du droit implique que chaque concept soit exprimé par un mot particulier dans chaque langue. De même que le découpage de la réalité varie d'une langue à l'autre, celui de la réalité juridique varie d'un système juridique à l'autre. Ainsi, chaque système choisit tel mot au détriment de tel autre pour exprimer et définir un concept juridique particulier.

Traiter de la terminologie juridique en usage en Algérie nécessite une approche pluridisciplinaire, ce pays maghrébin, au croisement de l'Europe et de l'Afrique, possédant un héritage culturel et civilisationnel très complexe. Si l'arabe est le véhicule par excellence du droit musulman, la recherche terminologique dans cette langue doit alors prendre en compte la manière de concevoir et de conceptualiser la réalité juridique dans cette culture mais aussi intégrer les réalités socioculturelles du locuteur algérien. Nous nous inscrivons donc dans le cadre de la socioterminologie, non sans affinités avec l'approche culturelle présentée par DikiKidiri car, quelle que soit la communauté, " c'est sa vision du monde qui détermine sa façon de classer, d'ordonner, de nommer et de catégoriser tout ce qu'elle perçoit ou conçoit, y compris sa propre identité ". (Diki-Kidiri, 2000)

\section{Qu'est-ce que la socioterminologie ?}

Selon Maria Teresa Cabré :

" [...] la terminologie ne prétend pas fournir une explication des termes selon les principes de la linguistique théorique, ni décrire le comportement terminologique des spécialistes; elle cherche seulement à proposer des éléments théoriques et des principes qui régissent le dépouillement, la sélection et le classement des termes propres des domaines dans le but de les normaliser. " (Cabré, 77)

La terminologie est la discipline qui étudie les vocabulaires spécialisés et en analyse les conditions d'utilisation dans un cadre normalisé. Pour l'École de Rouen, il s'agit non seulement d'étudier les conditions d'utilisation, mais aussi les mécanismes de création du lexique spécialisé 
par les membres de la communauté sociale. L'analyse socioterminologique est donc une analyse terminologique sous un angle sociolinguistique. En effet, étudiant les termes dans leur dimension discursive, la socioterminologie " procède avant tout d'une attitude descriptive " (Gaudin, 152) en rupture avec l'attitude prescriptive de la terminologie traditionnelle d'obédience wüstérienne(1) (consultation d'experts, normalisation, etc.). Ce faisant, elle relève aussi de la glottopolitique, laquelle permet de réfléchir à la pluralité des forces qui influent sur les pratiques langagières et à la dimension conflictuelle relative aux enjeux dénominatifs dans des sphères d'activité. Les termes sont ainsi appréhendés sous l'angle de leur acception au sein des pratiques sociales et langagières des locuteurs.

Par ailleurs, à la partition langue générale/langue spécialisée, la socioterminologie préfère la notion de continuum. Les termes ne sont donc plus appréhendés comme des unités décontextualisées, mais au sein d'une praxis déterminée qui pèse sur leur interprétation. Ainsi, la notion de sphère d'activité (par ex. celle se rapportant au droit) est préférée à celle de domaine qui sous-tend l'idée d'une communauté langagière stable. En outre, les termes en usage sont analysés à partir d'un corpus représentatif des pratiques langagières attestées au sein de la sphère d'activité concernée et construit sur la base d'un certain nombre de critères d'homogénéité et de contrastes. Les corpus ne sont pas de simples recueils indifférenciés d'énoncés.

"La socioterminologie s'occupe de termes et, par là, ne peut faire l'économie de l'usage qui en est fait ", affirme Valérie Delavigne, avant d'ajouter que cette discipline opère " un déplacement de l'objet d'étude par rapport à l'approche terminologique traditionnelle, dans la mesure où ce sont les discours spécialisés dans leur globalité qui deviennent objet d'intérêt, et plus seulement les termes. " (Delavigne, 38). L'approche socioterminologique nécessite la collecte de termes dans leur contexte discursif, oral ou écrit. Elle rend compte de la dimension sociale de la terminologie et place la culture au cœur de sa démarche. C'est le choix adopté ici s'agissant des termes juridiques extraits du Code de la famille et du Code de la nationalité qui forment notre corpus écrit.

\section{La comparaison des terminologies}

En l'occurrence, il s'agit de deux nomenclatures provenant des mêmes textes, le Code de la famille et le Code de la nationalité, respectivement en arabe et en français. Le double objectif de cette comparaison est de voir si la terminologie en arabe permet de rendre compte efficacement des réalités du domaine du droit et de cerner les différents rapports existant entre les notions dans cette langue et en français.

(1) II s'agit de la théorie terminologique d'Eugen Wüster qui se développe entre les deux guerres sous l'influence du positivisme du cercle de Vienne. 
Dans les travaux terminologiques bilingues ou multilingues, la comparaison des nomenclatures s'effectue d'abord et surtout à partir de celle des notions, car " ce n'est pas une équivalence de dénominations que l'on recherche d'abord, mais une équivalence de notions " (Rondeau, 33). Une notion renvoyant à une réalité unique est souvent exprimée par des dénominations différentes lorsque l'on considère des langues distinctes. Dans une recherche sur deux langues, l'analyse et la comparaison des notions offrent trois situations : 1) les situations d'équivalence, 2) les situations de quasi-équivalence et 3) les situations d'absence de notions dans l'une ou l'autre des deux langues. Nous présenterons ces cas successivement.

\section{L'équivalence des notions}

En terminologie bilingue, certains termes des langues considérées dans un même domaine de spécialité " affichent une identité complète de sens et d'usage " (Dubuc, 55). L'équivalence de notions décrit alors cette situation où une notion dans une langue donnée correspond exactement à une notion dans une autre langue. Ainsi, entre l'arabe et le français on trouve plusieurs termes équivalents dans le domaine du droit, notamment dans notre corpus. Exemples: " nationalité " en langue française est rendu par jinsīa (الجنسية) en langue arabe. II s'agit de deux termes équivalents. C'est aussi le cas d'" attribution de la nationalité " et de manh al-jinsīa (تجنّس) (تنح الجنسية), de " naturalisation " et de tajannus.

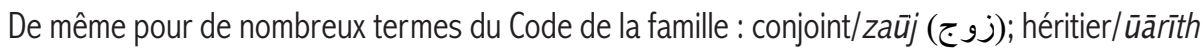
(وارث) ; dهبة); destament/ ūașīa (وصيّة); contrat/'aqd (عقد), etc.

\section{La quasi-équivalence des notions}

On parle de quasi-équivalence quand une notion dans une langue donnée est plus étendue qu'une notion dans une autre langue. Robert Dubuc (Dubuc, 55) emploie le terme de correspondance, que nous lui empruntons, pour décrire cette situation dans laquelle un terme ne recouvre que partiellement le champ sémantique d'un autre, entraînant des " disparités de sens ", ou dans laquelle il se situe à un " niveau de langue différent de son homologue de l'autre langue ", avec dans ce cas des "disparités d'usage ". Dans les disparités de sens, on distingue, à la suite de Guy Rondeau, deux cas : soit il faut plusieurs termes dans une langue donnée pour correspondre à un terme dans une autre langue ; soit la notion dans une langue donnée ne recouvre que partiellement la notion exprimée dans l'autre langue.

L'exemple du mot arabe hadāna est, à cet égard, très intéressant. Le traducteur juridique algérien prend en effet la précaution de ne pas toujours le traduire par "garde ". II est ici confronté à un concept propre à la culture juridique musulmane qui dépasse de loin celui de 
la " garde " en langue et culture juridique françaises. C'est pourquoi dans le texte français, on retrouve quasi systématiquement la translittération haḍāna entre parenthèses à côté du mot " garde ". C'est aussi le cas du terme français " mariage ", utilisé tantôt comme équivalent du mot zaūāj (زواج), tantôt comme équivalent du mot nikāh (نكاح), et du terme wali, tantôt rendu en français par " tuteur ", tantôt par la translittération " wali ".

\section{Notions absentes dans l'une des deux langues}

Dans certains cas, la notion juridique est inexistante dans l'une des deux langues. C'est ainsi le cas de fātihaā, (l'ouverture, en français) qui est la translittération du mot arabe (الفاتحة), le nom d'une sourate du Coran ; et de khula', translittération du terme arabe خلع) non traduit dans l'énoncé en langue française, et qui désigne l'action accomplie par une épouse qui se sépare délibérément de son conjoint. C'est aussi le cas de al muchtaraka, al mubahala, al minbariya, etc., notions complexes traitant de l'héritage, sans équivalents en langue française.

Le traducteur algérien est souvent confronté à ce problème de non-équivalence entre les notions lorsqu'il tente un transfert d'une culture juridique à une autre. Cela semble se confirmer dans la traduction du Code de la famille algérien qui, beaucoup plus que les autres textes de loi (le Code civil ou le Code pénal, par exemple), est très fortement pénétré par le droit musulman (Bessadi, 44).

\section{Traduction à visée informative ou à visée pragmatique ?}

Dans l'accomplissement de sa tâche, le traducteur doit aussi s'interroger sur la visée de la traduction : informative ou bien pragmatique, c'est-à-dire ayant les effets juridiques de l'original. Si la finalité de l'opération traduisante est d'obtenir un texte ayant valeur légale, elle posera davantage de problèmes au traducteur dans ses choix terminologiques. Le degré de difficulté sera donc déterminé, dans une large mesure, par l'usage auquel est destinée la traduction.

Officiellement, c'est au texte rédigé en langue arabe qu'il appartient de créer le droit en Algérie. La version en langue française ne serait donc rédigée qu'à titre informatif. En réalité les choses sont beaucoup plus compliquées et le débat opposant ceux qui soutiennent que l'arabe est la langue source et ceux qui soutiennent l'inverse est toujours en cours.

Deux thèses peuvent ainsi être avancées pour expliquer les nombreux cas de non-équivalence dans la traduction des énoncés des Codes de la famille et de la nationalité algériens ainsi que le recours excessif à la translittération dans la traduction en langue française de certains termes juridiques arabes tirés du droit musulman. La première est celle d'une double rédaction en lieu et place d'une entreprise de traduction. Autrement dit, les deux textes seraient 
rédigés par deux équipes distinctes, l'une francophone, l'autre arabophone, et les deux énoncés seraient ainsi destinés à deux lectorats distincts. Cette hypothèse est néanmoins peu probable étant donné qu'il s'agit de textes très sensibles, fondateurs de l'État algérien. La seconde postule que, contrairement aux autres textes officiels de l'État algérien, les Codes de la famille et de la nationalité algériens seraient les seuls à avoir été d'abord rédigés en langue arabe, puis traduits en français, uniquement pour information, seul le texte en langue arabe faisant foi et ayant force de loi.

Certes, dans le cas des textes officiels en Algérie, il est difficile, en l'absence de communication officielle sur le sujet par les instances concernées au sommet de l'État, de savoir s'il s'agit d'une traduction à visée informative ou d'une traduction à visée pragmatique. Cette question, comme celle maintes fois évoquée de la langue initiale de rédaction de ces mêmes textes, fait l'objet d'un blackout complet et, partant, d'un débat récurrent entre spécialistes en Algérie.

\section{Conclusion}

Nous constatons divers problèmes dus au fait que certains termes ne sont pas tout à fait équivalents, ou que certaines notions n'ont tout simplement pas de dénominations dans l'une des deux langues. Dans une optique socioterminologique et face aux vides dénominationnels en arabe et en français, il serait bon de proposer des dénominations pour les situations juridiques constituant des réalités dans les deux langues, afin qu'elles soient mieux prises en charge, et pour un meilleur transfert de connaissances entre elles dans le domaine du droit.

Cette recherche de nouveaux termes devra tenir compte de la structure et du fonctionnement des deux langues, mais aussi de la vision du monde de la communauté qui les utilise. Cet enrichissement, qui doit naître d'un besoin réel, peut se faire de façon naturelle, par l'évolution de la langue et l'action des locuteurs face à des besoins précis de communication dans le domaine du droit. II peut aussi se faire de manière " artificielle ", à des fins pratiques, par le biais d'une collaboration entre spécialistes de la langue (linguistes et terminologues) et spécialistes du droit en Algérie.

bessadinourredine@gmail.com

Nourredine Bessadi est doctorant en sciences du langage au département de français de l'université Abderrahmane Mira de Béjaia, Algérie. 


\section{Bibliographie}

BESSADI Nourredine, "Le traducteur juridique algérien face à la difficulté de traduire certains termes juridiques arabes ", Alternative francophone [en ligne], 17 déc. 2010, p. 40-48. https://ejournals.library.ualberta.ca/index.php/af/article/view/9532/pdf

CABRÉ Maria Teresa, Terminologie : théorie, méthode et applications, Les presses de l'Université d'Ottawa, Armand Colin, 1998.

DELAVIGNE Valérie, " Approche socioterminologique des discours du nucléaire ", Meta, vol. 40, $n^{\circ} 12,1995$, p. 308-319.

DIKI-KIDIRI Marcel, dir. "Une approche culturelle de la terminologie ", dans Terminologies nouvelles, $n^{\circ} 21$, juin 2000.

DUBUC Robert, Manuel pratique de terminologie, 3e édition, entièrement revue et remise à jour, Linguatech, Brossard (Canada), 1992.

GAUDIN François, "L'apparition de la socioterminologie : une position épistémologique " in Où en sont les sciences du langage 10 ans après ?Éd. ASL, 1992, p. 151-160.

RONDEAU Guy, Introduction à la terminologie, 2e éd., réimpression, Gaëtan Morin, Boucherville (Canada), 1984. 